http://jmscr.igmpublication.org/home/ ISSN (e)-2347-176x ISSN (p) 2455-0450 crossref DOI: https://dx.doi.org/10.18535/jmscr/v10i1.13

\author{
Journal Of Medical Science And Clinical Research

\title{
Microperimetry and SD-OCT study comparative study in individuals with diabetes without diabetic retinopathy and healthy controls
}

\author{
Authors \\ Dr Preeti Rawat ${ }^{1}$, Dr Divya Khandelwal ${ }^{2}$, Dr Varun Upadhyay ${ }^{3^{*}}$ \\ ${ }^{1}$ Professor, MGM Medical College \& MY Hospital, Indore (MP) \\ ${ }^{2}$ Resident Doctor, MGM Medical College \& MY Hospital, Indore (MP) \\ ${ }^{3}$ Senior Resident, Shyamal Shah Medical College, Rewa (MP) \\ *Corresponding Author \\ Dr Varun Upadhyay \\ Senior Resident, Shyamal Shah Medical College, Rewa (MP), India
}

\section{Introduction}

Diabetic retinopathy (DR) mainly in Type 2 diabetics is one of the major causes of permanent vision loss in working population. ${ }^{1}$ In DR, before significant visual loss patient may suffer other disturbances such as waviness, blurring, relative scotoma which is not assessed by routine examination. Therefore, suspicion and detection of early signs of neuro degeneration could be beneficial in preventing vision loss and deciding appropriate therapeutic approach in the management of diabetic retinopathy which is achieved by integration of retinal details by fundus related perimetry (microperimetry) \& correlating it with the precise location of oedema or thickness by OCT. ${ }^{2}$

Spectral domain OCT is a newer-generation highresolution OCT with the advantages of high-speed data acquisition, three-dimensional reconstruction of acquired retinal images, and improved visualization of retinal architecture, layer by layer whereas microperimetry offers the possibility to obtain an exact fundus related quantification of retinal sensitivity. Therefore, this study will help us to identify, among diabetic patients, a population at higher risk of developing vision threatening retinopathy. ${ }^{3}$

\section{Aims \& Objectives}

- To elucidate thickness changes in neurosensory retina in the macular area, using SD OCT \& correlate with functional loss on fundus -related microperimetry, in patients with Diabetes \& no DR.

- To determine whether neuronal dysfunction is an early sign of DR

\section{Material \& Method}

Study Design- Prospective clinical observational study

Sample Population- Patients coming to MYH OPD, Indore

\section{Inclusion Criteria}

Previously diagnosed DM without DR changes \& controls were subjects with no history of DM

\section{Exclusion Criteria}

- Cataract (NS Grading>2, PSC, Posterior Polar) 
- Coexisting ocular diseases

- History of intraocular surgery except uneventful cataract surgery and postoperative period.

- Ocular trauma

- Increased IOP

- Neurological disease causing visual field defects

- Disease causes optic neuropathy

- Uveitis

- Hypertension

\section{Patient Assessment}

Done by taking Complete history.

Examination of subjects is done by assessing: BCVA

Slit lamp biomicroscopy
IOP measurement

Fundoscopy

Microperimetry with SLO-MP using modified 102, central 14 degrees \& Retinal thickness in the macular region measured with OCT using 3D retinal topography (central $3 \mathrm{~mm}$ ).

\section{Measurement \& Analysis of Macular thickness}

Central $3 \mathrm{~mm}$ of the macula is analyzed using $3 \mathrm{D}$ retinal topography module of SD-OCT.

Central circle (1mm diameter circle), the outer circle/zone covers an area from $1 \mathrm{~mm}$ diameter to $3 \mathrm{~mm}$ diameter. The outer circle/zones are divided into sub regions marked as: Superior, Temporal, Inferior, Nasal regions.

Each of the regions \& sub regions displays average thickness \& volume.

Mean of all 5 regions was calculated and noted as shown in figure below

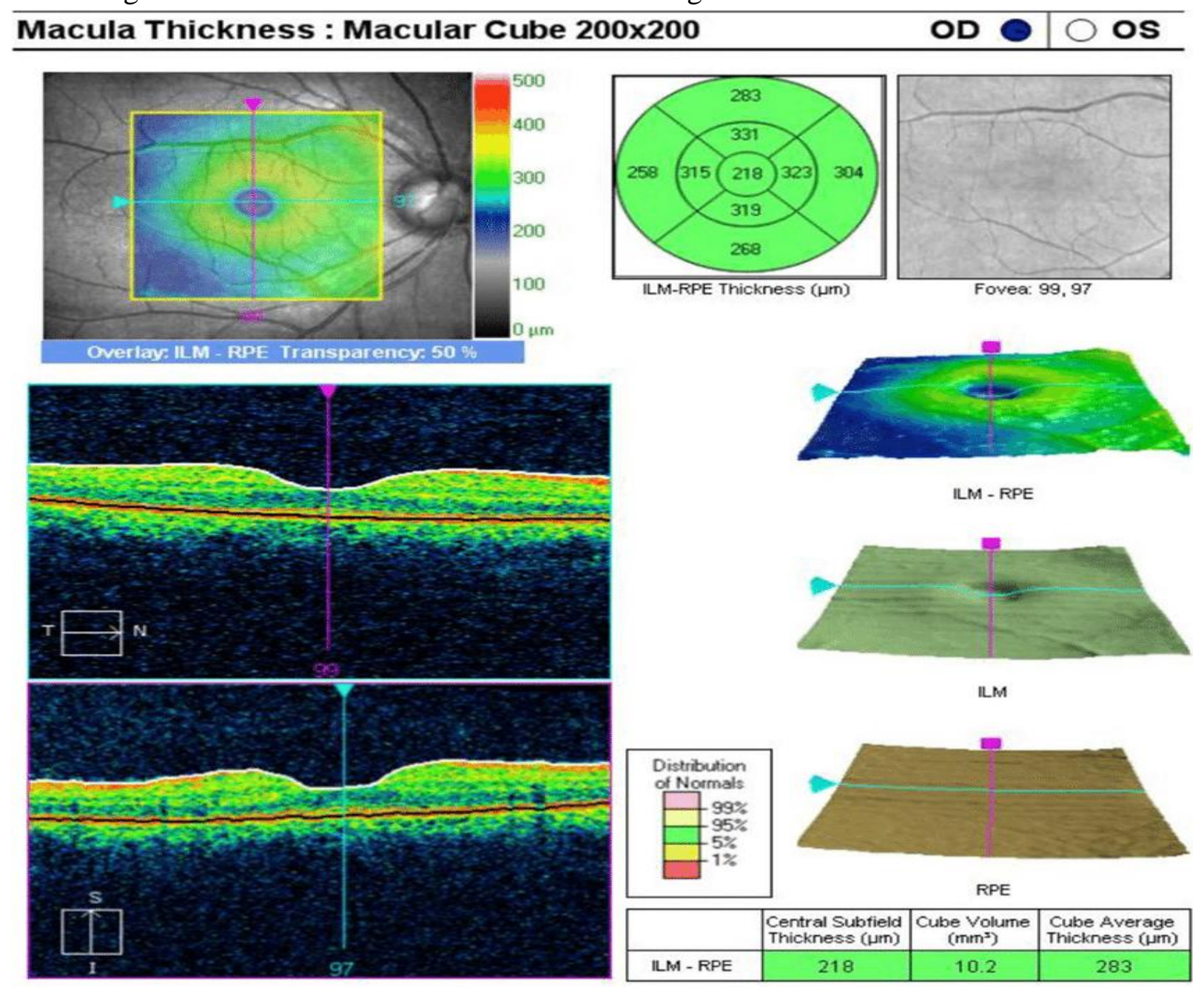


Retinal threshold sensitivity \& Microperimetry

Retinal threshold sensitivity was determined using modified 10-2 pattern in central 14 degrees of macula.

Stimulus size: Goldmann-3; Strategy-4-2; duration-200ms

Sensitivity is measured in terms of DECIBELS (db).

Statistical Analysis was done using 2014 Graph pad Software (at www.graphpad.com)

\section{Observations}

30 eyes in each group i.e. study \& control group were studied.

Total 41 subjects were studied (22 in study group \& 19 in control).
Mean age of study \& control group was $52.8 \&$ 51.9 years respectively

Mean thickness in study group was $251.3+/-1.055$ microns \& in control group was $253.83+/-1.384$ microns. No statistical difference was found amongst the two groups as $\mathrm{p}$ value $=0.1508$ on unpaired t-test.

Mean threshold retinal sensitivity was $10.253+/-$ $\mathrm{db}$ in study group \& $12.330+/-\mathrm{db}$ in control group. Difference was found to be statistically significant $(\mathrm{p}<0.0001)$.

Mean perimetry test score was $534.00+/-6.38 \mathrm{db}$ in study group \& $644.3+/-\mathrm{db}$ in control group. Difference was found to be statistically significant $(\mathrm{p}<0.0001)$.

Table 1 Showing comparison of mean macular thickness (microns) of two groups

\begin{tabular}{|l|c|c|}
\hline & Study Group & Control Group \\
\hline Mean & 251.3 & 253.83 \\
\hline Standard deviation & 5.78 & 7.58 \\
\hline Standard error & 1.055 & 1.384 \\
\hline Range & $240.2-262.8$ & $241.4-268.6$ \\
\hline
\end{tabular}

Table 2 Showing comparison of threshold retinal sensitivity (decibels) in the two group

\begin{tabular}{|l|c|c|}
\hline & Study Group & Control Group \\
\hline Mean & 10.253 & 12.330 \\
\hline Standard deviation & 0.655 & 0.546 \\
\hline Standard error & 0.120 & 0.100 \\
\hline Range & $8.9-11.0$ & $11.5-13.3$ \\
\hline
\end{tabular}

\section{Discussion}

This study was performed at a tertiary care hospital, Indore

Findings in our study was consistent with the similar study performed by Mehmet Demir et al (April 2013), titled "Central Macular Thickness in patients with Type 2 DM without clinical retinopathy" on 124 eyes of 62 subjects \& compared the central $1 \mathrm{~mm}$ Macular thickness with that of 120 eyes of healthy subjects. ${ }^{4}$

Threshold retinal sensitivity in study group \& healthy subjects were found to be $10.23+/-0.12$ \& $12.33+/-0.10$ respectively. This is suggestive of early neuronal dysfunction or neuronal loss in the retina. This finding was consistent with the study conducted by Verma et al (September 2009) titled "Is neuronal dysfunction an early sign of DR? Microperimetry \& spectral domain OCT study in individuals with diabetes, but no DR" performed on 39 patients in each group. ${ }^{5}$

Electrophysiological studies shows alterations in the retina, both early in type 2 diabetics \& after a long duration of both type $1 \&$ type 2 . Some of the alterations are present before any vascular retinopathy is detectable on fundus photography, indicating that neuronal damage may develop parallel with vascular changes.

Microperimetry scores appear to correlate well with disruptions in the inner segment -outer segment (IS-OS) functional layer. 


\section{Summary \& Conclusion}

In this study 2 groups were formed \& studied.

Subjects under both the groups underwent complete ophthalmological examination, macular thickness assessment by SD-OCT topography, central 14 degrees microperimetry with the microperimetry module on the same machine.

3 main parameters were collected from each subject i.e. Mean macular thickness, threshold retinal sensitivity \& microperimetry test score at the end of the complete evaluate. These parameters were compared amongst the 2 groups.

Significant difference in the mean threshold retinal sensitivity \& test score is highly suggestive of early functional loss of the retina. This loss prior to appearance of DR in study group indicates presence of Neuronal dysfunction or neuronal tissue loss.

This concept of Neuronal dysfunction sets up a new paradigm in the management of DR, \& also shifts the focus from therapeutic to preventive approach towards patients of diabetes \& hence improves the patient care.

\section{Reference}

1. Hyva rinen et al., 1983

2. Sharma et al.,2005; Owsley and Sloane, 1987; Midena, 2006

3. Carpineto P, Ciancaglini M, Di Antonio L, Gavalas C, Mastropasqua L.(Retina. 2007 Jan;27(1):21-9).

4. Mehmet Demir et al J Ophthalmol.2013; 2013; 767931. Published online Apr 3, 2013. Doi: $10.1155 / 2013 / 767931$

5. Verma A, Rani PK, Raman R, Pal SS, Laxmi G, Gupta M, Sahu C, Vaitheeswaran K, Sharma T. Eye (Lond). 2009 Sep;23(9);1824-30. Doi 10.1038/eye.2009.184. Epub 2009 Jul 24 